\title{
Avanços no rastreamento mamográfico e o manejo das pacientes com câncer de mama
}

\author{
Advances in mammographic screening and management of patients with breast cancer
}

Avances en el cribado mamográfico y el tratamiento de pacientes con cáncer de mama

Maira Dorighetto Ardisson ${ }^{1}$, Ynara Olivier Junger², Débora Freitas Miranda², Georgia Maciel da Silva Brito $^{2}$, Emanuella Vieira de Siqueira Botelho ${ }^{2}$, Sonia Alves Gouvea ${ }^{1}$, Taynan Nunes Ribeiro ${ }^{3}$, Sergio Wilson Alves Pereira², Neuzimar Rodolfo Serafim², Rodrigo Alves Faria1*.

\section{RESUMO}

Objetivo: Revisar sobre os métodos de rastreamento por tomossíntese e por mamografia convencional, bem como, sua aplicação no contexto clínico e manejo das pacientes com câncer de mama. Revisão bibliográfica: $O$ câncer de mama é a maior causa de mortalidade e comorbidade entre as mulheres podendo também acometer os homens. Portanto, o rastreamento é imprescindível para o controle e identificação precoce dessa doença, consequentemente, a mamografia configura-se como principal método. No entanto, dentre as mulheres submetidas a mamografia, existe uma parcela consistente que possuem parênquima mamário de maior densidade, fator no qual pode impedir a visualização precoce de lesões na mama resultando em diagnóstico falso negativo pela mamografia tradicional. Diante disso, a tomossíntese melhor tecnologia possibilitando clareza na imagem e o diagnóstico com mais solidez de forma precoce nesses casos, uma vez que é capaz de diminuir a sobreposição do tecido fibroglandular, permitindo aumento na detecção das lesões. Considerações finais: Visando o aprimoramento diagnóstico em consonância com o desenvolvimento tecnológico, a tomossíntese é uma técnica eficaz de detecção de neoplasias mamárias e pode ser associada a mamografia possibilitando o rastreamento mais eficaz.

Palavras-chave: Mamografia, Neoplasias da mama, Tomossíntese digital de mama, Detecção precoce de câncer, Saúde da Mulher.

\section{ABSTRACT}

Objective: To review the methods of screening by tomosynthesis and conventional mammography, as well as their application in the clinical context and management of patients with breast cancer. Bibliographic Review: Breast cancer is the major cause of mortality and comorbidity among women and may also affect men. Therefore, screening is essential for the control and early identification of this disease, consequently, mammography is the main method. However, among women undergoing mammography, there is a consistent proportion that have a higher density breast parenchyma, a factor in which it may prevent the early visualization of breast lesions resulting in a false negative diagnosis by traditional mammography. Therefore, tomosynthesis is a better technology, enabling clarity of the image and a more solid diagnosis at an early stage in these cases, since it is able to reduce the overlap of fibroglandular tissue, allowing an increase in the detection of lesions. Final Considerations: Aiming at improving diagnosis in line with technological development, tomosynthesis is an effective technique for detecting breast neoplasms and can be associated with mammography, enabling more effective screening.

Keywords: Mammography, Breast neoplasms, Digital Breast Tomosynthesis, Early cancer detection, Women's Health.

${ }^{1}$ Universidade Federal do Espírito Santo (UFES), Vitória - ES. *E-mail: rodrigo.ufes@hotmail.com

${ }^{2}$ Hospital Evangélico de Vila Velha (HEVV), Vila Velha - ES.

${ }^{3}$ Centro Capixaba de Oncologia (CECON), Vitória - ES. 


\section{RESUMEN}

Objetivo: Revisar los métodos de cribado por tomosíntesis y mamografía convencional, así como su aplicación en el contexto clínico y manejo de pacientes con cáncer de mama. Revisión bibliográfica: El cáncer de mama es la principal causa de mortalidad y comorbilidad entre las mujeres y también puede afectar a los hombres. Por tanto, el cribado es fundamental para el control e identificación precoz de esta enfermedad, por lo que la mamografía es el método principal. Sin embargo, entre las mujeres que se someten a una mamografía, hay una proporción constante que tiene un parénquima mamario de mayor densidad, un factor en el que puede prevenir la visualización temprana de las lesiones mamarias, lo que resulta en un diagnóstico falso negativo mediante la mamografía tradicional. Por tanto, la tomosíntesis es una mejor tecnología, permitiendo claridad de imagen y un diagnóstico más sólido en una etapa temprana en estos casos, ya que es capaz de reducir la superposición de tejido fibroglandular, permitiendo un aumento en la detección de lesiones. Consideraciones finales: con el objetivo de mejorar el diagnóstico de acuerdo con el desarrollo tecnológico, la tomosíntesis es una técnica eficaz para detectar neoplasias de mama y puede asociarse con la mamografía, lo que permite un cribado más eficaz.

Palabras clave: Mamografía, Neoplasias mamarias, Tomosíntesis digital de mama, Detección precoz de cáncer, Salud de la mujer.

\section{INTRODUÇÃO}

O câncer de mama apresenta-se como uma doença heterogênea a nível molecular, patológico e clínico, com diferentes graus de agressividade tumoral e alto potencial metastático. De acordo com a Internacional Agency for Research on Cancer (IARC) e a plataforma Global Cancer Observatory (GLOBOCAN), é estimado que até o ano de 2040 haja 3,5 milhões de novos casos e aproximadamente 1 milhão de óbitos, consolidando o câncer de mama como a doença mais incidente e com a maior mortalidade entre as mulheres em todo o mundo, independente do Índice de Desenvolvimento Humano (IDH). Estas taxas são mais altas no continente asiático e na Europa, seguidas pela América do Norte, América Latina e Caribe e são mais baixas na África e na Oceania (HENRI C, et al., 2016; IARC, 2020).

As neoplasias da mama também afetam a população masculina, no entanto, é considerado raro, representando entre $0,8 \%$ a $1 \%$ do total desta modalidade, ou seja, para cada 100 novos casos de câncer de mama nas mulheres, apenas 1 caso em homens será diagnosticado. No Brasil, segundo o Instituto Nacional do Câncer (INCA), as estimativas para o ano de 2020 são de 66.280 novos casos e 17.572 mortes, favorecendo assim, o expressivo prejuízo emocional, econômico e o social das pacientes e de seus familiares (LITIÈRE S, et al., 2012; HENRI C, et al., 2016; INCA, 2019 e 2020).

O comportamento dinâmico do câncer está em constante transformação, em razão da sua individualização orgânica e da extrema heterogeneidade tumoral associada à presença de fatores de risco conhecidos e não conhecidos, tais como, idade avançada, gravidez tardia, uso de terapia de reposição hormonal, mutações de BRCA1 e BRCA2, ter tido câncer de mama no passado, etilismo, obesidade e sedentarismo podem potencializam as chances de acometimento (CARDOSO CF, et al., 2018; FISHER B, et al., 2002; SIEGEL $\mathrm{RL}$, et al., 2020).

Os estudos de Henri C, et al. (2016) e Siegel RL, et al. (2020) atribuem a elevada incidência do câncer de mama às mudanças habituais da sociedade oriundas dos diversos processos de industrialização. Em vista disso, é necessário não apenas da conscientização dos meios de prevenção, através de mudanças no estilo de vida, como também da compreensão dos métodos atuais disponíveis para a consolidação do rastreio e do diagnóstico adequados. Para tanto, é imprescindível a atenção do governo às modalidades acessíveis à população que pode fazer a diferença entre o diagnóstico precoce e com melhor prognóstico clínico ou o diagnóstico tardio com pior prognóstico de sobrevida.

Essa relação já era explorada desde o século passado e continua até os dias atuais devido a compreensão do auxílio na contenção da doença em seu estágio inicial, e, consequentemente na obtenção positiva da sobrevida livre de recidiva devido a manutenção do tamanho do tumor, seu acometimento linfático e a 
presença de metástases consolidando na ampliação da sobrevida global. Alguns estudos mostraram que atrasos superiores a três meses sem intervenção aumentam a probabilidade de morte quando comparados ao menor tempo de espera, e que os pacientes com maior duração dos sintomas são diagnosticados em estágios mais avançados da doença com tumores maiores, ocasionando o aumento da mortalidade, principalmente nos países em desenvolvimento (UNGER-SALDAÑA K, et al., 2015; UNGER-SALDAÑA K e INFANTE C, 2009; PARDO RC e WIESNER CC, 2014; WELCH HG, et al., 2016; SHARMA K, et al., 2012; GONZALO ZR, et al., 2010).

No intuito de evitar estes desfechos clínicos, as evidências científicas apontam para os benefícios do rastreamento mamográfico na redução da mortalidade por câncer de mama, considerando que esta detecção pode aumentar em até $30 \%$ a taxa de cura. Mesmo ao longo dos anos e com os refinamentos obtidos a mamografia digital de campo total 2D, ainda se consolida como o exame mais indicado para o rastreio do câncer de mama, atuando como uma importante ferramenta na acurácia diagnóstica, principalmente em mulheres mais jovens, abaixo dos 49 anos, mesmo apresentando limitações, como a qualidade inferior da imagem na sobreposição dos tecidos e das estruturas da mama, por exemplo, em mulheres com parênquima mamário com maior densidade (FREER PE, et al., 2017; GILBERT FJ, et al., 2016).

Várias das limitações ao uso da mamografia 2D foram superadas a partir de 2011 ao surgir à técnica mamográfica 3D, conhecida por Tomossíntese Digital da Mama com a opção de eliminar a sobreposição de tecidos e com isso, obter melhorias no campo de visualização conduzindo à sua melhor interpretação. Estes avanços comparativamente forneceram a qualidade necessária à tomossíntese digital da mama na prática de triagem, considerando que, o exame convencional 2D não evidencia alguns tumores às vezes encobertos por tecidos fibroglandulares acarretando em resultados falsos negativos (GILBERT FJ, et al., 2016; MYERS ER, et al., 2015; GARCÍA-ALBENIZ X, et al., 2020; KIM YJ, et al., 2018).

Com isso, várias evidências sobre a capacidade de detecção da tomossíntese mamária como uma modalidade de rastreamento do câncer de mama têm se estabelecido a cada dia, porém, os estudos científicos sobre esta tecnologia e o manejo dos pacientes diagnosticados necessitam ser esclarecidos. Portanto, no intuito de participar desse processo de construção, o objetivo desse estudo é revisar sobre os métodos de rastreamento por meio da tomossíntese digital da mama em 3D e pela mamografia convencional 2D, bem como, sua aplicação no contexto clínico e manejo das pacientes com câncer de mama (HADJIPANTELI A, et al., 2019; SIEGEL RL, et al., 2020; FREER PE, et al., 2017).

\section{REVISÃO BIBLIOGRÁFICA}

No intuito de viabilizar o diagnóstico para o câncer de mama, o rastreamento da população obteve seu desenvolvimento com base em várias evidências científicas e a sua implementação nos sistemas públicos ou suplementares de saúde se consolida um importante marco na redução da mortalidade dos pacientes acometidos devido a sua capacidade de detectar o desenvolvimento do tumor antes das manifestações clínicas quando realizados rotineiramente. Estes avanços ao longo dos anos forneceram subsídios fundamentais para a evolução dos métodos, como, a transição da antiga tela de filme para a realização da mamografia digital e, recentemente a utilização da tomossíntese digital da mama, demonstrando que esta técnica reduz as limitações e melhorar a precisão da imagem em comparação com a mamografia convencional (GARCÍA-ALBENIZ X, et al., 2020; PLEVRITIS SK, et al., 2018).

Embora alguns estudos possam descrever a sua superioridade em relação à mamografia digital 2D, são questões que ainda dividem opiniões, principalmente sobre a possibilidade de inserir a tomossíntese como padrão clínico para o rastreamento em larga escala nas situações de alterações mamárias. 0 desenvolvimento e a acurácia destes novos métodos são imprescindíveis para minimizar os resultados falsospositivos e falsos-negativos oriundos de uma imagem bidimensional. A estrutura da mama é tridimensional, por isso, os tecidos normais quando sobrepostos propiciam densidades diferentes acompanhados de texturas diferentes caracterizando imagens com aparências distintas e complexas que consolidam em muitas situações suspeitas devido a assimetria ou distorções, fazendo com que imagens adicionais ou até mesmo a solicitação para a realização de biópsias sejam exigidas (CARDOSO CF, et al., 2018; HOOLEY RJ, et al., 2017). 
A tomossíntese digital mamária, além de ser um exame bem tolerado, permite a visualização tridimensional do tecido mamário, ou seja, permite uma imagem ampla sem as possibilidades de sobreposição de tecidos ou de dificuldades encontradas devido a sua densidade. O diagnóstico do câncer de mama atualmente no mundo é realizado em sua grande parcela por triagem de rotina, e neste contexto, por ser essencial, sua realização proporciona a redução da mortalidade ao possibilitar o tratamento precoce devido a sua capacidade de detectar nódulos e microcalcificações não palpáveis no exame físico (SIMON S, et al., 2019; SILVA GA, et al., 2014; PLEVRITIS SK, et al., 2018).

Embora a mortalidade por câncer de mama tenha diminuído consideravelmente desde a década de 1980, acredita-se que os avanços terapêuticos estejam mais relacionados a esta redução do que propriamente a realização do rastreamento pelos pacientes. Ademais, a determinação desse risco por meio dos testes genéticos pode reduzir a incidência do câncer de mama, seja por quimioterapia preventiva, cirúrgica profilática ou mesmo possibilitar o tratamento precoce se o rastreamento for iniciado em pacientes com idades mais jovens, porém, seu custo elevado impossibilita o acesso à grande parte da população (GILBERT FJ, et al., 2016; WELCH HG, et al., 2016).

Ainda não existe um consenso sobre as porcentagens exatas de risco ao longo da vida para desenvolvimento do câncer de mama, por isto, determinar uma categoria no intuito de identificar mulheres com risco médio, moderado e alto pode trazer benefícios consistentes a esta população. Os principais fatores usados possuem como base o histórico pessoal ou familiar de câncer de mama, câncer de ovário, câncer de tubas ou peritônio, a associação entre as mutações de BRCA 1 e 2, possuir mutação patogênica no paciente ou em algum parente, ter realizado biópsia de mama anterior indicando lesão de alto risco ou ter realizado radioterapia no tórax entre 10 e 30 anos de idade (RENCK DV, et al., 2014; HADJIPANTELI A, et al., 2019; CONANT EF, et al., 2020).

$\mathrm{O}$ acesso ao rastreamento precoce e a estas tecnologias possuem elevado valor para os pacientes com risco associado ao desenvolvimento do câncer de mama, em virtude da maior eficácia ao tratamento prévio, $\mathrm{e}$, consequentemente na redução da mortalidade. Ao determinar o risco, segue o encaminhamento para a modalidade mais adequada e a frequência de realização do rastreamento. Sabe-se que alguns tipos de câncer se apresentam ocultos à mamografia, portanto, para serem detectados são necessários a realização de outros exames de imagem ou pelo exame físico, principalmente quando estes tumores se apresentam como nódulos. Aproximadamente metade das mulheres que são submetidas à mamografia de rastreamento possuem parênquima mamário de maior densidade, tornando comum outros encaminhamentos posteriores (WELCH HG, et al., 2016; PARDO RC e WIESNER CC, 2014).

As principais sociedades brasileiras que tratam sobre o câncer de mama, recomendam a mamografia anual para mulheres a partir dos 40 anos de idade, visando o diagnóstico precoce e a redução da mortalidade neste público, no entanto, esta medida difere das orientações fornecidas pelo Ministério da Saúde, que a preconizam bianualmente, a partir dos 50 anos, excluindo mulheres entre 40 e 49 anos de idade, responsáveis por cerca de 15 à 20\% dos casos de câncer de mama (PLEVRITIS SK, et al., 2018; SIEGEL RL, et al., 2020; FREER PE, et al., 2017).

Contudo, o rastreamento realizado pelo sistema público de saúde entre as mulheres de alto risco, possibilita a identificação precoce, mesmo existindo divergências sobre a faixa etária. Diversos estudos coadunam que em países desenvolvidos, ocorre a redução de $44 \%$ na mortalidade entre a faixa etária dos 40 aos 69 anos pelo rastreio precoce, resultado bem distante do ideal no Brasil. Somado a este entrave, apenas $35 \%$ das mulheres com indicação para a mamografia o fazem regularmente a cada 2 anos, sendo, uma das dificuldades o acesso ao exame ou a disponibilidade dos mesmos em sua região de residência (ACR, 2020; MIGOWSKI AR, et al., 2018 e 2019).

A idade avançada nas mulheres é um fator de risco relevante no Brasil para o desenvolvimento do câncer de mama, para tanto, em 2019, a expectativa de vida se consolidou nos 80 anos de idade em comparação aos homens com 73 anos, coincidindo com a expressiva incidência de câncer de mama a partir dos 75 anos. Em decorrência do elevado número de mortes nesta faixa etária, estimados em $26 \%$, métodos preventivos personalizados podem reduzir substancialmente o diagnóstico tardio. Assim, a maioria dos estudos 
prospectivos e ensaios clínicos randomizados contemplam mulheres acima da sétima década de vida. Atualmente o que se preconiza é a discussão e individualização da decisão a ser tomada, priorizando o rastreamento em mulheres com expectativa de vida maior que 75 anos (URBAN LA, et al., 2017; GARCÍAALBENIZ X, et al., 2020).

Entretanto, autores brasileiros consideram que a falta de acesso aos serviços de saúde é uma das mais importantes causas da progressão da doença e da redução da sobrevida global, para tanto, alguns estudos observacionais questionam os benefícios do rastreamento em comparação com as baixas taxas de adesão, concluindo que a realização de tratamentos dispensáveis e a exposição à radiação somados com a saúde mental debilitada e as condições socioeconômicas deficientes das famílias acometidas são fatores relevantes e necessita ser esclarecido (SIMON S, et al., 2019).

A clara evidência científica do rastreamento na redução da mortalidade em alguns estudos é discutida positivamente, e os benefícios fornecidos pela triagem são substanciais, bem acima de qualquer efeito negativo. Porém, mesmo neste contexto, são necessários estudos com o uso de métodos mais eficientes permitindo resultados mais rápidos na triagem convencional, considerando que o câncer de mama ainda carrega taxas de mortalidade expressivas somadas a pior qualidade de vida nas pacientes com doença avançada. Em alguns estudos sobre a mamografia convencional, os resultados falso-negativos eram consolidados em virtude do tamanho do tumor, no entanto, a relação entre tumores maiores e a presença de metástases está bem estabelecido na literatura, bem como, sua associação aos piores desfechos (MIGOWSKI AR, et al., 2019; HENRI C, et al., 2016; KREITER E, et al., 2014).

Convencionalmente o padrão-ouro para triagem sem sinais ou sintomas é a mamografia $2 \mathrm{D}$, mesmo possuindo sensibilidade que varia entre $80 \%$ a $90 \%$ e especificidade entre $50 \%$ a $70 \%$, somados ao custo relativamente baixo, consiste na aquisição de imagem da mama por meio de feixes de raio $\mathrm{X}$ de baixa energia e com capacidade de gerar imagens de elevado contraste e resolução. Por exame são realizados no mínimo quatro imagens com o objetivo de visualizar a anatomia da mama e detectar sinais de doença, sendo alterações nos nódulos mamários, assimetrias, distorções e calcificações como os principais (UNGERSALDAÑA K, et al., 2015; WELCH HG, et al., 2016).

No intuito de auxiliar o diagnóstico precoce, a evolução tecnológica mamográfica por meio do desenvolvimento de sofisticadas técnicas de computação permitiram que a tomossíntese mamária tivesse este destaque na clínica. Para a realização deste exame, a mama é posicionada e comprimida de forma semelhante à mamografia, no entanto o tubo de raio $X$ necessita se deslocar em um arco 15 graus, obtendo aproximadamente 15 imagens da mama, que são reconstruídas em imagens tomográficas tridimensionais fatiadas em $1 \mathrm{~mm}$ de espessura por meio de software computacional, minimizando a sobreposição dos tecidos (SKAANE P, et al., 2018; CONANT EF, et al., 2020; TABÁR L, et al., 2011).

A tomossíntese deve ser realizada em associação com a mamografia digital ou com uma imagem bidimensional sintetizada, visto que, com esta configuração obteve êxito na detecção do câncer de mama em até $40 \%$ em relação à mamografia isolada, além de, reduzir a taxa de reconvocação das pacientes para realização de estudos adicionais em $15 \%$. Seu principal benefício em relação à mamografia consiste na redução da sobreposição do tecido fibroglandular, permitindo aumento na detecção, caracterização morfológica e na localização das lesões, além destes pontos positivos, permite maior detecção de cânceres de pior prognóstico e aumento significativo na detecção do carcinoma lobular invasivo, devido a produção de imagens em 3D, proporcionando benefícios mais expressivos a ponto de diminuir a necessidade da realização de algumas biópsias (HOUSSAMI N e MIGLIORETTI DL, 2016; KIM YJ, et al., 2018; BERNARDI D, et al., 2016).

Esse método foi introduzido em programas de rastreamento do câncer de mama em alguns países, e, está sendo considerado para introdução na prática clínica de rastreio em vários outros. Atualmente, não há padronização para esta implementação na triagem de rotina, e, em muitos casos, há a falta de orientação oficial sobre o uso ideal de cada sistema disponível comercialmente. As principais desvantagens da tomossíntese resultam do incremento da dose de radiação ionizante, maior tempo de interpretação pelo médico e custo do equipamento elevado quando comparado ao mamógrafo digital (BERNARDI D, et al., 2016; MENDES LC, et. al.,2018; SIMON S, et al., 2019; GONZALO ZR, et al., 2010). 
Atualmente na prática clínica a utilização da tomossíntese não fica restrita apenas a alguns grupos de pacientes, podendo ser utilizado em qualquer idade, sendo particularmente útil nas pacientes mais jovens que apresentam parênquima mamário de maior densidade. Devido a qualidade da imagem, as margens e o tamanho do tumor são melhor avaliados, bem como, a localização das lesões são evidenciadas com mais nitidez, por isso, alguns especialistas sugerem que a tomossíntese pode ser preferida à mamografia padrão, embora este não seja o exame padrão (SKAANE P, et al., 2018; GARCÍA-ALBENIZ X, et al., 2020).

Em contrapartida, nos Estados Unidos, a National Comprehensive Cancer Network (NCCN) atualizou suas diretrizes para mulheres com risco médio de câncer de mama, adicionando "considere" o rastreamento de tomossíntese anual a partir dos 40 anos, e para mulheres de risco médio, não havendo a necessidade de triagem suplementar adicional com outras modalidades quando a tomossíntese é realizada como o exame de triagem inicial (SKAANE P, et al., 2018; HADJIPANTELI A, et al., 2019; CHONG A, et. al., 2019).

A detecção de tumores ainda ocultos de estágio T1 ou N0 pela tomossíntese é mais favorável. Por mais que atualmente já exista evidências de que a tomossíntese mamária tem um papel importante no rastreamento e na detecção do câncer de mama precoce por fornecer informações relacionadas ao delineamento de pequenas lesões e por minimizar interpretações contraditórias sobre os tecidos sobrepostos, outros estudos randomizados não fornecem evidências consistentes de sua melhora no diagnóstico inicial apontando para o fator heterogeneidade devido as variações dependendo do estudo e do ambiente de triagem. Assim, ainda é necessário conhecer os seus benefícios na redução da mortalidade e quais os grupos de risco e faixa etária poderiam ser beneficiados (CONANT EF, et al., 2020; CARDOSO CF, et al., 2018; ZACKRISSON S, et al., 2018; GILBERT FJ, et al., 2016).

\section{CONSIDERAÇÕES FINAIS}

Compreender os benefícios da detecção precoce do câncer de mama possibilitou avanços tecnológicos essenciais aos métodos de rastreio, evidenciando que a tomossíntese digital da mama melhora as medidas de detecção inicial do câncer com menores taxas de reconvocação. O exame de rastreio ideal é aquele que possui alta sensibilidade e especificidade, que seja seguro e possua relação custo-benefício, reduzindo os transtornos, custos da investigação do falso-negativo e seu impacto na redução da mortalidade, ou seja, elementos deficientes no Brasil, onde, a falta de acesso aos serviços de saúde determina a progressão de novos casos e a necessidade de tratamentos mais tóxicos. Novos estudos devem se concentrar na avaliação de triagem repetida com tomossíntese, elucidando os resultados a longo prazo, incluindo o efeito nas taxas de câncer de intervalo.

\section{AGRADECIMENTOS E FINANCIAMENTO}

Agradecemos à Fundação de Amparo à Pesquisa e Inovação do Espírito Santo (FAPES) e ao Centro de Ensino e Pesquisa do Hospital Evangélico de Vila Velha (HEVV) pelo auxílio financeiro concedido.

\section{REFERÊNCIAS}

1. AMERICAN COLLEGE OF RADIOLOGY - ACR. Practice parameter for communication of diagnostic imaging findings. ARC, 2020.

2. BERNARDI D, et al. Breast cancer screening with tomosynthesis (3D mammography) with acquired or synthetic $2 \mathrm{D}$ mammography compared with 2D mammography alone (STORM-2): a population-based prospective study. The Lancet Oncology, 2016; 17: 1105-1113.

3. CARDOSO CF, et al. Characterization of male breast cancer: results of the EORT C 10085/TCMRC/BIG/NACMG International Male Breast Cancer Program. Annals of Oncology, 2018; 29: 405-417.

4. CHONG A, et al. Digital Breast Tomosynthesis: Concepts and Clinical Practice. Radiology, 2019; 292:1-14.

5. CONANT EF, et al. Five Consecutive Years of Screening with Digital Breast Tomosynthesis: Outcomes by Screening Year and Round. Radiology, 2020; 295(2): 285-293.

6. FISHER B, et al. Vinte anos de acompanhamento de um estudo randomizado comparando mastectomia total, mastectomia e mastectomia mais irradiação para o tratamento de CM invasivo. N Engl J Med, 2002; $347: 1233$.

7. FREER PE, et al. Clinical implementation of synthesized mammography with digital breast tomosynthesis in a routine clinical practice. Breast Cancer Res Treat, 2017; 166(2):501-509. 
8. GARCÍA-ALBÉNIZ X, et al. Continuação dos exames anuais de mamografia e mortalidade por câncer de mama em mulheres com mais de 70 anos. Ann Intern Med, 2020; 172: 381.

9. GILBERT FJ, et al. Tomossíntese digital de mama (TCD): uma revisão das evidências para uso como ferramenta de rastreamento. Clin Radiol, 2016; 71 (2): 141-150.

10. GLOBOCAN - Global Cancer Observatory: Taxas estimadas de mortalidade em mulheres padronizada por idade em 2018, em todo o mundo. Câncer de Mama. Disponível em: https://gco.iarc.fr/today/online-analysis-multi. Acesso em: 17 Maio 2020.

11. GONZALO ZRD, et. al. Impacto dos fatores prognósticos e preditivos do câncer de mama na Unidade Provincial de Oncologia, Matanzas. Electronic Medical Rev, 2010; 32 (5).

12. HADJIPANTELI A, et al. The role of digital breast tomosynthesis in breast cancer screening: a manufacturer-and metrics-specific analysis. Cancer Manag Res, 2019; 11: 9277-9296.

13. HENRI C, et al. The Role of Biomarkers in Decreasing Risk of Cardiac Toxicity after Cancer Therapy. Biomark Cancer, 2016; 8: 39-45.

14. HOOLEY RJ, et al. Avanços na tomossíntese digital da mama. AJR Am J Roentgenol, 2017; $208: 256$.

15. HOUSSAMI N, MIGLIORETTI DL. Tomossíntese digital da mama: um admirável mundo novo de exames de mamografia. JAMA Oncol. 2016; 2 (6): 725-727.

16. INSTITUTO NACIONAL DE CÂNCER - INCA. Estimativa 2020: Incidência de Câncer no Brasil. MS / INCA / Coordenação de Prevenção e Vigilância / Divisão de Vigilância e Análise de Situação, 2020.

17. INSTITUTO NACIONAL DE CÂNCER - INCA. José Alencar Gomes da Silva: A situação do CM no Brasil: síntese de dados dos sistemas de informação. / Instituto Nacional de Câncer José Alencar Gomes da Silva. INCA, $2019 ; 85$.

18. INTERNATIONAL AGENCY FOR RESEARCH ON CANCER - IARC / Globocan / Agência Internacional de Pesquisa sobre Câncer 2020 Disponível em: https://gco.iarc.fr/tomorrow/home. Acesso em: 17 maio 2020.

19. KIM YJ, et al. Comparação da tomossíntese mamária digital em duas visualizações com a mamografia digital em três visualizações em um ambiente de triagem simulada. Acta Radiol, 2018; 60 (9) 1-8.

20. KREITER E, et al: Cancro da mama: Tendências na incidência internacional em homens e mulheres. Br J Cancer, 2014; $110: 1891-1897$.

21. LITIĖRE S, et al. Terapia conservadora da mama versus mastectomia para CM em estágio I-II: acompanhamento de 20 anos do estudo randomizado fase 3 do EORTC 10801. Lancet Oncol, 2012; 13: 412.

22. MENDES LC, et al. Revisão sistemática: mamografia digital vs tomossíntese mamária digital - comparação de desempenho. Anais do simpósio de Engenharia Biomédica. Even3, 2018.

23. MIGOWSKI, ARN, et al. Diretrizes para detecção precoce do $\mathrm{CM}$ no Brasil. III - Desafios à implementação. Cadernos de Saúde Pública, 2019; 34(6): e00046317

24. MIGOWSKI, ARN, et al. Diretrizes para detecção precoce do CM no Brasil. II - Novas recomendações nacionais, principais evidências e controvérsias. Cadernos de Saúde Pública, [online], 2018; 34 (6): e00074817.

25. MYERS ER, et al. Benefícios e danos do rastreamento do câncer de mama: uma revisão sistemática. JAMA, 2015; 314: 1615.

26. PARDO RC, WIESNER CC. Anuário Estatístico 2014. Instituto Nacional de Cancerologia, 2014; $12: 118$.

27. PLEVRITIS SK, et al. Association of Screening and Treatment with Breast Cancer Mortality by Molecular Subtype in US Women, 2000-2012. JAMA, 2018; 319: 154.

28. RENCK DV, et al. Equidade no acesso ao rastreamento mamográfico do câncer de mama com intervenção de mamógrafo móvel no sul do Rio Grande do Sul, Brasil. Cadernos de Saúde Pública. 2014; 30(1): 88-96.

29. SHARMA K, et al. Uma revisão sistemática das barreiras ao tratamento do câncer de mama em países em desenvolvimento, resultando na apresentação tardia do paciente. J Oncol, 2012; 1-8.

30. SIEGEL RL, et al. Estatísticas do Câncer, 2020. CA Cancer. J Clin, 2020; 70: 7.

31. SILVA GA, et al. Acesso à detecção precoce do câncer de mama no Sistema Único de Saúde: uma análise a partir dos dados do Sistema de Informações em Saúde. Cad Saúde Pública, 2014; 30(7): 1537-50.

32. SIMON S, et al. Characteristics and prognosis of stage I-III breast cancer subtypes in Brazil: The AMAZONA retrospective cohort study. The Breast, 2019; 44: 113-119.

33. SKAANE $P$, et al. Performance of breast cancer screening using digital breast tomosynthesis: results from the prospective population-based Oslo Tomosynthesis Screening Trial. Breast Cancer Res Treat, 2018;169: 489-496.

34. TABÁR L, et al. Swedish Two-County Trial: Impact of Mammographic Screening on Breast Cancer Mortality during 3 Decades. Radiology, 2011; 260: 658-663.

35. UNGER-SALDAÑA $\mathrm{K}$, et al. Atraso no sistema de saúde e seu efeito no estágio clínico do câncer de mama: estudo multicêntrico. Câncer, 2015; 121 (13): 2198-2206.

36. UNGER-SALDAÑA K, INFANTE C. Atraso de cuidados médicos para câncer de mama sintomático: Uma revisão da literatura. Public Health Mex, 2009; 51 (supl. 2): S270-85

37. URBAN LABD, et al. Recomendações do Colégio Brasileiro de Radiologia e Diagnóstico por Imagem, da Sociedade Brasileira de Mastologia e da Federação Brasileira das Associações de Ginecologia e Obstetrícia para o rastreamento do câncer de mama. Radiol Bras [online], 2017; 50: 244-249.

38. WELCH HG, et al. Tamanho do tumor de câncer de mama, sobrediagnóstico e eficácia do rastreamento de mamografia. N Engl J Med, 2016; 375: 1438.

39. ZACKRISSON S, et al. One-view breast tomosynthesis versus two-view mammography in the Malmö Breast Tomosynthesis Screening Trial (MBTST): a prospective, population-based, diagnostic accuracy study. The Lancet Oncology, 2018;19: 1493-1503. 